\title{
Deliberação moral de enfermeiros frente à internação por ordem judicial
}

Moral deliberation of nurses facing hospitalization by court order

Deliberación moral de enfermeras sobre la hospitalización por orden judicial

\section{Kely Regina da Luz ${ }^{\text {I }}$ Mara Ambrosina de Oliveira Vargas ${ }^{\mathrm{II}}$, Edison Luiz Devos Barlem ${ }^{\mathrm{III}}$, Dulcinéia Ghizoni Schneider ${ }^{\mathrm{IV}}$, Franciele Budziareck das Neves ${ }^{\mathrm{v}}$}

\begin{abstract}
Resumo: Objetivo: analisar os problemas éticos vivenciados por enfermeiros intensivistas em situações de internação por ordem judicial na perspectiva da deliberação moral. Método: pesquisa qualitativa entre janeiro e dezembro de 2016, mediante entrevistas semiestruturadas com 42 enfermeiros intensivistas das regiões Sul e Sudeste do Brasil. Apreciada por meio da Análise Textual Discursiva. Resultados: a análise resultou em três categorias que apresentam como conteúdo a prioridade da deliberação, o sofrimento moral dos enfermeiros e a advocacia do enfermeiro. Considerações finais: a judicialização da saúde traz alterações significativas nas relações sociais e institucionais. Os enfermeiros devem perceber que, frente às diferentes possibilidades de tomadas de decisão diante dos problemas éticos, valores morais importantes encontram-se em conflito.

Descritores: Cuidados críticos; Ética em enfermagem; Decisões judiciais; Enfermagem; Doente terminal
\end{abstract}

Abstract: Aim: to analyze the ethical problems experienced by intensive care nurses in situations of hospitalization by judicial order in the perspective of moral deliberation. Method: qualitative research carried out between January and December 2016, through semi-structured interviews with 42 intensive care nurses from South and Southeast regions of Brazil. Interpreted through Discursive Textual Analysis. Results: the analysis resulted in three categories that present in their content the priority of deliberation, the moral suffering of nurses and the nurse's advocacy. Final considerations: health judicialization brings significant changes in social and institutional relations. Nurses must realize that, facing the different possibilities of decision-making in the face of ethical problems, important moral values are in conflict.

Descriptors: Critical Care; Ethics, Nursing; Judicial Decisions; Nursing; terminally ill

\footnotetext{
${ }^{\text {I }}$ Enfermeira. Doutora em Enfermagem e Mestre em Enfermagem pela Universidade Federal de Santa Catarina - UFSC. Especialista em Enfermagem em Terapia Intensiva e em Nefrologia. Enfermeira da Unidade de Terapia Intensiva Cardiológica do Hospital de Clínicas de Porto Alegre - HCPA. Porto Alegre, RS. Brasil. kelydaluz@gmail.com. ORCID: 0000-0003-1616-5515

II Enfermeira. Doutora em Enfermagem. Professora do Departamento de Enfermagem e do PEN/UFSC. Florianópolis, SC, Brasil. E-mail: ambrosina.mara@ufsc.br ORCID: 0000-0003-4721-4260

${ }^{\text {III }}$ Enfermeiro. Doutor em Enfermagem. Docente do Curso de Graduação e Pós-Graduação de Enfermagem da Universidade Federal do Rio Grande. Rio Grande, RS, Brasil. E-mail: ebarlem@gmail.com ORCID: 0000-0001-6239-8657

IV Enfermeira. Doutora em Enfermagem. Professora do Departamento de Enfermagem da UFSC. Florianópolis, Santa Catarina, Brasil. E-mail: dulcineiags@gmail.com ORCID: 0000-0002-4842-2187

` Enfermeira. Mestre em Ciências. Doutoranda do PEN/UFSC, Florianópolis, SC, Brasil. E-mail: fran.bnvs@gmail.com. ORCID: 0000-0002-20159153
} 
Resumen: Objetivo: analizar los problemas éticos vividos por las enfermeras de cuidados intensivos en situaciones de hospitalización por orden judicial, desde la perspectiva de deliberación moral. Método: investigación cualitativa, desarrollada entre enero y diciembre de 2016, por medio de entrevistas semiestructuradas, con 42 enfermeras intensivas de las regiones sur y sureste del Brasil. Los datos fueron analizados por análisis textual discursiva. Resultados: del análisis emergió tres categorías que presentan el contenido como prioridad de la deliberación, el sufrimiento moral de las enfermeras y la defensa de la enfermera. Consideraciones finales: la judicialización de la salud presenta cambios significativos en las relaciones sociales e institucionales. Las enfermeras deben observar que, en relación a las diferentes posibilidades de la toma de decisiones sobre problemas éticos, valores morales importantes están en conflicto.

Descriptores: Cuidados Críticos; Ética en Enfermería; Decisiones Judiciales; Enfermería; Enfermo Terminal

\section{Introdução}

O direito à saúde é considerado um direito universal, social e humano, pertencendo ao rol daqueles relacionados ao mínimo existencial devido ao que representa para a vida e sobrevivência de todo ser indivíduo. ${ }^{1}$ No Brasil, a demanda populacional requer que o Sistema Único de Saúde (SUS) seja eficiente e realmente disponível, para que, no momento que os indivíduos dele necessitem, este esteja presente. A teoria diverge da realidade neste aspecto, sendo ressaltada a insuficiência de insumos no que concerne ao atendimento populacional. Neste contexto, surge no Brasil um movimento em que o indivíduo já não recorre diretamente ao SUS para a efetivação de seu direito, mas sim, ao Poder Judiciário, a fim de garantir os seus direitos por meio da tutela jurisdicional. ${ }^{2}$

O crescimento das demandas judiciais na saúde é tema de grande relevância, tanto para o sistema de saúde, quanto para o sistema judiciário. ${ }^{3}$ No entanto, identificar os limites da atividade judicial e as possibilidades de controle sobre a atividade política é uma tarefa árdua e controversa. ${ }^{4} \mathrm{O}$ litígio pelo direito à saúde, no Brasil, torna clara a disputa de diferentes atores dos setores público e privado no processo de judicialização da saúde. Essas ações proporcionam o acesso a milhares de pessoas, ainda que provisoriamente, gerando realidades sociomédicas multifacetadas, além de importantes desafios administrativos e fiscais que podem aumentar as 
desigualdades no fornecimento de serviços de saúde, como o acesso aos leitos de Unidade de Terapia Intensiva (UTI). ${ }^{5}$

A internação em UTI possui critérios específicos, os quais têm como finalidade a priorização do atendimento no processo de triagem, ou seja, a internação dos pacientes que mais se beneficiarão do tratamento intensivo. Porém, quando o acesso aos serviços de saúde ocorre com a intervenção do Poder Judiciário, estas observâncias que disciplinam o acesso à saúde são ignoradas e, consequentemente, podem comprometer a organização legal do SUS. ${ }^{6}$ Enquanto alguns pacientes têm acesso por meios próprios aos tratamentos necessários para a recuperação das doenças que ameaçam sua vida, a maioria deve contar com a proteção e o acesso a que tem direito, mas que são limitados pelos recursos escassos e as fragilidades do sistema, apelando para judicialização da saúde..$^{5-6}$

Internações por ordem judicial demandam do enfermeiro que atua na UTI questionamentos sobre como deve agir nestes casos, suscitando posicionamentos e deliberações éticas complexas, com o enfrentamento de conflitos, pois, muitas vezes, o atendimento que presta é diferente daquele que gostaria de conceder, o que vai lhe causando sofrimento. ${ }^{3} \mathrm{~A}$ responsabilidade ética individual é o coração da competência clínica para todos que cuidam de pessoas doentes. Apesar dos avanços obtidos na prevenção e no tratamento de doenças, é possível constatar, de forma paradoxal, diversos fracassos na prática clínica, sobretudo no que concerne ao profissional de saúde lidar com fenômenos subjetivos da pessoa que necessita de cuidado. Logo, os profissionais de enfermagem mostram-se vulneráveis a crises de valores e queda da qualidade do seu trabalho, tendendo a enfrentar situações problemáticas relacionadas à internação por ordem judicial na UTI, quanto às tomadas de decisão em relação aos seus fazeres com implicações éticas para si, para os pacientes, para a equipe e para a instituição hospitalar. $^{7}$ 
Enfermeiros precisam desenvolver uma competência ética, necessária para lidar com os problemas éticos no processo de cuidar de pacientes internados na UTI. Uma proposta que pode auxiliar enfermeiros a tomar decisões baliza-se na análise da situação, utilizando-se como ferramenta para a deliberação moral. Essa é uma ação complexa que está envolvida não só pelo conhecimento técnico-científico, mas também por elementos subjetivos, crenças, princípios e valores e, muitas vezes, a decisão tomada não constitui a melhor opção, quando avaliada sob outros aspectos que não os utilizados por quem analisou o conflito..$^{8-9}$

Este estudo sinaliza a importância de utilizar a deliberação moral, como ferramenta no processo de análise de situações e de tomada de decisões, na busca por soluções concretas e prudentes, já que possibilita que os enfermeiros evidenciem as lacunas na compreensão de um problema ético e percebam as diferenças de valores e deveres entre os envolvidos. Para responder a questão de pesquisa: quais são os problemas éticos vivenciados por enfermeiros intensivistas em situações de internação por ordem judicial na perspectiva da deliberação moral? Objetivou-se analisar os problemas éticos vivenciados por enfermeiros intensivistas em situações de internação por ordem judicial na perspectiva da deliberação moral.

\section{Método}

Pesquisa exploratória analítica, com abordagem de natureza qualitativa, desenvolvida com enfermeiros que trabalham em UTI adulto das regiões Sudeste e Sul do Brasil, totalizando 42 enfermeiros; o encerramento do número de participantes ocorreu pela repetição teórica dos dados. Essas regiões foram eleitas como locais de estudo por englobarem $68 \%$ dos leitos de UTI do país. Os critérios de inclusão foram: ser enfermeiro e desenvolver a prática assistencial em UTI adulto, independente de idade, sexo, horário de trabalho, procedentes de instituições públicas e privadas. 
A seleção dos participantes ocorreu mediante amostragem não probabilística, do tipo bola de neve; método usado por sua característica de ser aplicável quando o foco de estudo é um problema sensível, tratando de um tema particular, precisando do auxílio de pessoas bem informadas sobre o assunto. ${ }^{10}$ Assim, o ponto de partida do estudo aconteceu nas capitais dos sete Estados, formando sete "Bolas de Neve" simultâneas. O contato com o primeiro participante de cada capital ocorreu por meio da escolha pelas autoras da pesquisa, devido ao contato em rede com muitos colegas nesta área. O participante seguinte sucedeu-se pela indicação desse primeiro e assim por diante.

A coleta de dados ocorreu por meio de entrevistas presenciais, no dia, local e horário de preferência dos participantes, guiada por roteiro semiestruturado e com duração média de 45 minutos; e mediante o contato por e-mail, sendo possível responder as perguntas online, através de um link. O período de coleta transcorreu de janeiro a dezembro de 2016 por se tratar de uma busca intencional e para abarcar uma variedade de experiências profissionais, focadas na vivência da situação de inviabilidade de leito de UTI e de internação por ordem judicial em diferentes contextos hospitalares.

A análise ocorreu mediante elementos da Análise Textual Discursiva, organizando seus argumentos em torno de quatro focos: desmontagem dos textos; estabelecimento de relações; captura de um novo emergente; e recolocação dos achados - em um processo auto-organizado. ${ }^{11}$ Para organização e gerenciamento dos dados, utilizou-se o software ATLAS-Ti.

A pesquisa foi desenvolvida de acordo com os princípios éticos exigidos na Resolução n⿳o 466/2012 do Conselho Nacional de Saúde e obteve aprovação do Comitê de Ética em Pesquisa com Seres Humanos do Centro de Pesquisas Oncológicas no dia 03 de outubro de 2014, sob o Certificado de Apresentação para Apreciação Ética nº 36739514.2.0000.5355. Para garantir o anonimato, os participantes foram codificados com as letras que identificam os seus Estados, 
seguido do número cardinal na ordem em que aconteceram as entrevistas em cada local, idade, tempo de formação e período de atuação em UTI.

\section{Resultados}

Diante dos discursos analisados, foram identificadas três categorias: 1) "A escolha de Sofia"? a deliberação sobre quem será priorizado 2) O sofrimento moral vivenciado pelo enfermeiro diante da internação por ordem judicial na UTI e 3) A advocacia do enfermeiro diante das demandas da sociedade na luta pela vida através dos avanços da tecnologia.

\section{"A escolha de Sofia"? a deliberação sobre quem será priorizado}

Utilizou-se a expressão “A Escolha de Sofia” por ser entendida como o sinônimo de uma decisão quase impossível de ser tomada. Situação vivenciada pelos enfermeiros deste estudo diante dos dilemas éticos, no que se refere à a decisão de "liberar" um paciente "menos" grave ou talvez que não tenha condições de receber alta e admitir o paciente que vem por demanda judicial, o que impõe um processo de deliberação moral. A percepção da existência de dilemas éticos e o exercício da deliberação moral estão retratados nas falas abaixo:

$\grave{A} s$ vezes é dilema, mas penso que a maioria das vezes é problema. $A$ questão da saúde e do direito das pessoas é muito complexo, é como um cobertor que tapa de um lado e destapa do outro lado. (PR1, 35 anos, 10 anos de formada, há 8 anos na UTI)

A deliberação moral acontece, mesmo diante de algo imposto como a internação por ordem judicial, pois pode servir de base para a definição de quem sai da UTI:

Acho que é mais um dilema ético, porque se não tenho vaga, tenho um número de leitos definidos na UTI e se tiver que colocar alguém, terei que tirar outro. Quando tinha leito extra, era colocar mais um leito e pronto! A gente brigava e acatava a ordem do juiz porque tem que acatar. Tínhamos problemas de receber ordem judicial, não era uma questão de vaga, 
colocávamos um leito extra, estávamos acostumados a trabalhar com leito extra, mas o problema era de não ter respiradores. Então para receber um paciente e colocar no respirador teria que tirar outro do respirador, e aí quem decide isso?(RS2, 57 anos, 33 anos de formada, há 33 anos na UTI)

Os enfermeiros também revelam, em seus depoimentos, a angústia centrada no conhecimento da alta precoce e o reconhecimento da responsabilidade pelo paciente:

Vivenciei um caso de internação por ordem judicial que a unidade mesmo com leitos bloqueados por quantitativo de pessoal precisou admitir um paciente do interior. Neste caso o plantonista agilizou uma alta de um paciente pós-operatório imediato de neurocirurgia que ficou no CTI [centro de tratamento intensivo] menos de 12 horas. (MG4, 28 anos, 5 anos de formado, há 4 anos na UTI)

O problema é quando a UTI está em lotação máxima, entra um pedido de internação por ordem judicial e mesmo não existindo leito, precisamos conseguir um. (MG5, 34 anos, 1 ano de formada, há 1 ano na UTI)

\section{O sofrimento moral vivenciado pelo enfermeiro diante da internação por ordem judicial na unidade de terapia intensiva}

Esta categoria retrata o ambiente de saúde como local emocionalmente carregado para pacientes, famílias e provedores, criando terreno fértil para que pessoas discordem sobre as decisões quanto ao que é "certo" e o que é "moralmente correto". Logo, a natureza da prática de enfermagem pode ser um potencial gerador de sofrimento moral.

Além disso, a hospitalização em UTI constitui-se por si só em um evento vital, estressor e desestabiliza física e emocionalmente toda a família, tendo como gravante a negação de uma internação imediata, sendo que recorrer ao judiciário é a única saída para os pacientes e familiares para conseguirem vaga.

Paciente sem condições clínicas ser transferido da UTI para um local de cuidados considerado "cuidados intermediários" para desocupar o leito de UTI, você sabe que o paciente é investível, jovem, com condições de melhora e ter que transferi-lo para que outro tenha o direito de leito 
também é uma situação muito ruim, tudo que você aprende e acredita se vai junto com o paciente que foi transferido precocemente. (RJ3, 29 anos, 7 anos de formada, há 4 anos na UTI)

Ter uma unidade lotada com todos os pacientes sem condições de alta e não conseguir resolver é complexo. Entendo o problema do porque a pessoa precisou entrar com essa solicitação para conseguir internar, mas você tem uma unidade lotada que também não consegue simplesmente de uma hora para outra tirar alguém [...] É preciso trazer o paciente para dentro. (PR2, 52 anos, 30 anos de formada, há 20 anos na UTI)

\section{A advocacia do enfermeiro diante das demandas da sociedade na luta pela vida através dos avanços da tecnologia}

Diante das demandas judiciais não justificadas pela clínica, mas por expectativas familiares que estão à frente na luta pela vida de seu ente querido e temerosos pelo recurso de tratamento disponibilizado, na tentativa de proteção da dignidade do paciente a advocacia em saúde se faz necessária. Socialmente considerada um local pouco acolhedor e, frequentemente, associada à morte ou doença grave, e por outro lado, relacionada com atenção integral, recuperação e reencontro com a vida, a UTI necessita de profissionais prudentes, pois implica, necessariamente, a consideração dos valores, crenças e do projeto de vida de cada um, precisando o profissional perceber os diferentes sistemas de valores, padrões culturais, religiosos e familiares dos usuários de quem cuida.

Penso que o enfermeiro não deve questionar o paciente que dá entrada na UTI. Temos que tratar todo mundo igual, independente independente da maneira como chega. Para nós enfermeiros não vai mudar muita coisa, aliás, muda nada. (SC1,38 anos,13 anos de formado, há 1 ano na UTI)

Na UTI, o ser humano está exposto a uma multiplicidade de aparelhos sofisticados e complexos que podem determinar, por exemplo, os padrões ventilatórios, os sinais vitais e os 
9| Luz KR, Vargas MAO, Barlem ELD, Schneider DG, Neves FB

valores hemodinâmicos. Logo, há a preocupação com a forma de utilização das tecnologias como um fim em si mesmo, ao invés de um meio para assegurar a qualidade da assistência.

Observa-se que o progresso técnico-científico na saúde não somente aumentou a esperança de viver mais e melhor, mas também gerou situações dificílimas e complexas que trouxeram profundos questionamentos éticos e somos instigados a advogar de forma responsável, respeitando a vida e honrando a dignidade humana.

Paciente sem condições clínicas para cirurgia cardíaca de alta complexidade, família, embora orientada por toda a equipe, inclusive a enfermagem, atrás de ordem judicial, impôs o procedimento que resultou no óbito do paciente. (ES2, 54 anos, 28 anos de formada, há 27 anos na UTI)

Acredito que o enfermeiro que realmente advoga em favor do paciente, quando tem e utiliza os argumentos corretos, é capaz de indicar e convencer família e equipe sobre internação ou não na UTI sem a necessidade de apelar para decisão judicial, o que acho mais justo e ético do que forçar uma internação em detrimento das demais. (SP6, 33 anos, 8 anos de formada, há 7 anos na UTI)

A UTI possui uma dinâmica operacional e um ambiente bastante diferenciado de outros serviços do hospital. Adota-se uma série de critérios para a admissão e alta de paciente no atendimento intensivo; esses critérios foram criados para permitir o uso racional desses leitos com o objetivo de, assim, permitir que pacientes sejam mantidos ali somente quando seu estado de saúde exigir, podendo a imposição de uma internação por ordem judicial fragilizar tal processo.

Tive uma paciente que ficou internada por muito tempo, estava com alta e a família, mesmo de posses, não queria levar ela para casa. Essa internação prolongada, dificultou o atendimento de outras pessoas. Precisamos fazer a ordem judicial para que ela fosse embora. $O$ filho argumentou que ela sempre foi professora do Estado; agora o Estado cuide dela agora já que está precisando. (PR2, 52 anos, 30 anos de formada, há 20 anos na UTI) 
A dicotomia SUS e Convênio desperta inúmeras questões entre os profissionais de saúde e as pessoas que buscam o atendimento. A crescente tecnificação da saúde, com aparelhos, exames e medicamentos de última geração, aliada à ideologia da saúde perfeita e à consequente tendência cultural de identificar saúde com consumir produtos que vendem saúde, ocasionam um gradativo aumento de gastos induzidos por essa tendência que o orçamento público não conseguirá suprir.

Muitas pessoas não possuem condições financeiras para custear o melhor tratamento devido aos custos descomunais. Por isso, a advocacia deve ser exercida pelos profissionais, conforme as falas a seguir:

Nós temos pacientes daqui que são pacientes de convênio, e aqui tudo é SUS. E eles não são transferidos para hospitais conveniados porque não têm vaga, mas ele enquanto cidadão tem direito, pois ele é usuário do SUS, então eu não posso forçar o paciente que tem convênio a sair, ele está no direito dele. (RS2, 57 anos, 33 anos de formada, há 33 anos na UTI) Paciente mesmo com direito à internação em instituição privada porque tem convênio, nem sempre consegue vaga de UTI. Eu já vivenciei muito isso. Eles ficam esperando na emergência alguma situação e por vezes os familiares ameaçam colocar na justiça e colocam mesmo [já vivi isso de paciente pedir ajuda judicial parainternar em UTI mesmo com opção de convênio]. (PR1, 35 anos, 10 anos de formada, há 8 anos na UTI)

\section{Discussão}

A internação de paciente em UTI é precedida de condições críticas, presentes e potenciais, que colocam em risco a vida do ser humano. Em decorrência, o cuidado é voltado para os aspectos físico-orgânicos/orgânicos/biológicos, como controle e manutenção das funções vitais, com ênfase no uso de tecnologia e aplicação de conhecimento técnico-científico, visando à manutenção da vida. A emergência, a imediaticidade e a dramaticidade das situações vivenciadas na UTI fazem com que os problemas éticos, nesses locais, sejam frequentemente mais evidentes, tempestuosos e avultados. ${ }^{12}$ Nessa perspectiva, investiga-se até que ponto os 
enfermeiros conseguem deliberar? Destaca-se, aqui, a importância dessa prática, mesmo que não seja possível ser concretizada, para o desenvolvimento e fortalecimento da competência ética desse profissional.

Balizada nos resultados desse estudo, uma das primeiras questões abordada é a de justamente a enfermagem - e os demais profissionais -considerar que está diante de um problema ético. Neste caso, a equipe de enfermagem se aproxima do paciente para ajudá-lo no tratamento e com isso o vínculo entre eles se inicia. A dimensão ética do trabalho tende a passar despercebida por diversos profissionais, que, repetidamente, passam por questões éticas relevantes, contudo com dificuldade de identificar essas situações. ${ }^{13}$

Ainda, estudo sinaliza que, usualmente, diversas situações experienciadas pelos profissionais, são meramente identificadas como inconformidades institucionais e/ou problemas de relacionamento na equipe de enfermagem e de saúde. Isto é, elas são relacionadas com a administração, as chefias, os pacientes e os familiares e não como problemas ou conflitos morais. Surgem quando há heterogeneidade quanto ao entendimento diante de uma alguma situação, as quais não podem ser adequadamente comunicadas, compreendidas e resolvidas. ${ }^{14}$ Contudo, na contramão dessa premissa de não identificação do problema ético, os sentidos que emergem dos discursos dos participantes dessa pesquisa é de que as diferentes experiências com a internação por ordem judicial são imediatamente reconhecidas como uma questão ética.

Outro aspecto evidenciado nos discursos dos participantes direciona-se para situações que possibilitam a expressão do sofrimento moral diante da instabilidade dos pacientes que precisam internar na UTI e não têm vaga e dos que precisam sair e não têm condições. $\mathrm{Na}$ prática profissional, esses conflitos são vivenciados a partir de situações que inviabilizam disponibilizar tecnologia a serviço da vida, assim como o cumprimento do mandado judicial independente da concordância com o conteúdo da sentença. Para lidar com essas questões, os profissionais usam diferentes recursos de enfrentamento que podem ser positivos quando levam 
ao diálogo e à reflexão, e podem ser negativos, quando fazem o profissional aceitar e se conformar com a realidade, vivenciando sozinho situações éticas difíceis, sem apoio dos colegas ou da instituição e predispondo-se ao sentimento de sofrimento moral. ${ }^{15-17}$

A capacidade de tomar decisões perante questões éticas em enfermagem torna-se cada vez mais necessária, exemplificada nas falas dos participantes, o que está intimamente relacionado com os avanços da ciência e tecnologia, com a crescente complexidade das situações de saúde/doença e com uma maior autonomia dos enfermeiros. Logo, a deliberação moral se expressa na necessidade do profissional problematizar, questionar-se com as situações cotidianas, com as relações estabelecidas, considerandoo entendimento estabelecido pela e na coletividade. ${ }^{8}$

Em relação à decisão sobre quem entra e quem sai da UTI, uma pesquisa discute, entre outras questões, a ocupação de um leito de UTI por um paciente que não necessita mais de cuidados intensivos, relacionado às decisões de tratamento, mais especificamente a decisão de iniciar ou interromper a terapêutica. A natureza diferente do papel dos enfermeiros e médicos no cuidado do paciente leva a diferenças na forma como eles percebem e lidam com problemas éticos. Os resultados revelam que os entrevistados indicaram que há uma diferença clara entre os que participam do papel de decisão - os médicos, que têm perspectiva de "cura", suas indicações são expandidas por meio de um mecanismo de “o que é possível deve ser feito”, e aqueles que estão no papel do cuidado - os enfermeiros, que pela proximidade com o paciente e sua família podem fornecer informações valiosas sobre a carga de tratamento de um paciente, e podem proporcionar um contrapeso para o excesso de tratamento, sob alegação de que se preocupam porque os pacientes são muitas vezes submetidos à futilidade terapêutica. ${ }^{18}$

Diante disso, então, muitas vezes torna-se necessário e desejável o exercício de atividades e habilidades de advocacia em prol do paciente como um processo de deliberação moral. É uma atuação que vai além da intervenção clínica e tende a extrapolar os limites do setor saúde, sendo 
este um elemento especialmente munido de condições para identificar e compreender melhor a situação particular de vulnerabilidade apresentada pelo paciente e seu entorno familiar. ${ }^{19}$

Nesta direção, a advocacia também pode se manifestar quando o enfermeiro está diante da questão do paciente permanecer ou não em um local de tecnologia restrita, carência de estrutura física e recursos humanos necessários para manter a vida do paciente. ${ }^{7}$ A UTI apta para atender este tipo de paciente, muitas vezes, enfrenta a situação de já estar lotada e, mediante a ordem judicial, decidir sobre a quem dar prioridade de acesso a essas vagas limitadas, uma vez que a assistência e proteção da vida de uma pessoa pode significar a morte ou a complicação do estado de saúde de outra pessoa.

Um estudo, com 1.521 pacientes, ao relatar os dilemas éticos em UTI em relação ao último leito ou a ausência deste, determina a frequência de altas não programadas e avalia a relação entre a alta precoce e mortalidade hospitalar. No total da amostra, a taxa de mortalidade foi de 11,6\% (176 pacientes); destes que foram à óbito, 52 tiveram alta precoce, revelando que a alta não programada contribui significativamente para a mortalidade pós-UTI, e nisso está incluso o impacto da alta precoce de um paciente para permitir a admissão de outro. ${ }^{20}$

A judicialização da saúde desencadeia sucessivos dilemas que consolidam uma maneira vigente de experienciar o estresse cotidiano do trabalho nas instituições de saúde. Diante desses dilemas, tanto a participação direta como indireta do enfermeiro no processo de escolha entre quem sai e quem fica na UTI, de acordo com os resultados da pesquisa, expõe uma angústia centrada no conhecimento de que a alta precoce do paciente pode resultar em graves complicações. Assim, a deliberação moral, também, se articula ao pleno reconhecimento da responsabilidade pelo paciente, do zelo por sua integridade física, pelo seu tratamento, cuidado e bem-estar, conforme previsto no Código de Ética dos Profissionais de Enfermagem em seus princípios fundamentais, garantindo a qualidade dos serviços a serem prestados. 
Deliberação moral de enfermeiros frente à internação por ordem judicial | 14

Fortemente apoiada em tecnologia avançada e práticas especializadas, a medicina intensiva é responsável por uma rotina específica de cuidados intensivos e pela ampliação considerável da sobrevida de determinados pacientes. A UTI seria a porta para a imortalidade? Uma das dificuldades expressa nos discursos dos participantes reporta às condutas a serem tomadas com pacientes terminais, diante da pressão familiar que traz consigo o pensamento de que, enquanto há vida, há esperança. Nesse caso, a introdução de fatores econômicos, a tecnologia, a especialização da medicina, as mudanças no perfil dos pacientes e da sociedade, a presença do poder judiciário na saúde, além das expectativas e exigências dos pacientes têm interferido na relação profissional-paciente, o que leva a um processo de despersonalização, desumanização e desconfiança, causando frustração, desencanto e insatisfação. ${ }^{7}$ Logo, toda e qualquer deliberação moralconsideraque os eventos relacionados com a morte sãocomplexos e abrangem inúmeros problemas éticos e profissionais, os quais precisam ser trabalhados e discutidos à luz de princípios éticos que considerem a dignidade humana. ${ }^{21}$

A deliberação, por ser um comportamento ético, tem três momentos distintos do procedimento deliberativo: deliberação sobre os fatos, deliberação sobre valores e deliberação sobre os deveres. ${ }^{8}$ Esses valores subsidiam esse agir ético e são revelados nas falas dos participantes, traduzidos em: responsabilidade, autonomia, confiança, proteção, adequada comunicação, igualdade, empatia e sinergia.

Os princípios e valores da ética da enfermagem influenciam a tomada de decisão ética: os princípios éticos da autonomia, a beneficência, a não-maleficência e a justiça. Além dos fatores externos, como características organizacionais, limitações tecnológicas e/ou orçamentárias, falhas na comunicação com os demais profissionais, também os fatores individuais como, experiências, conhecimento e a comunicação, podem influenciar na tomada de decisão ética do enfermeiro. $^{22}$ 
Nos casos de planos de saúde no Brasil, os tribunais também foram convertidos em espaço importante para reivindicar os direitos dos consumidores, e como uma forma de exigir superação das lacunas entre o que está estipulado na política pública e o que foi implementado. Ao compreender o contexto social e cultural e os valores das pessoas e fundamentar-se no conhecimento científico, o enfermeiro encontra um caminho confortável para participar na tomada de decisão do usuário, e até mesmo, na construção da sua própria decisão ética e, através da deliberação moral, realizar julgamentos prudentes sobre os problemas vivenciados, inclusive diante de internação por ordem judicial. ${ }^{23}$

A importância da relação entre a equipe de saúde e a família é citada por diversos autores que consideram a comunicação adequada, o respeito e a compaixão com o paciente e seus familiares, como a chave determinante para a satisfação familiar. A comunicação em situações de terminalidade torna-se complexa em função dos fatores envolvidos e é parte fundamental do cuidado aos familiares. É, portanto, necessário que haja uma equipe multiprofissional especializada no atendimento deste paciente em todas as dimensões, garantindo-lhe o bemestar e respeito à sua dignidade. Porém, nestas circunstâncias, a relação médico-família enfrenta momentos cruciais, pois ambos devem enfrentar a gravidade da doença e os limites do tratamento, a despeito de todo aparato farmacológico e tecnológico disponíveis. ${ }^{24}$

\section{Considerações finais}

A judicialização da saúde traz alterações significativas nas relações sociais e institucionais; o Poder Público não pode negar o direito à saúde, por ser um direito de todos. Consequentemente, a sociedade, o Judiciário e o Estado em si não podem fechar os olhos para os pacientes que ingressam na Justiça e negar-lhes o atendimento médico de que necessitam. Portanto, emerge um dilema e uma escolha dramática. 
A solução não está presente desde o princípio e, portanto, a questão não está na eleição entre duas ou mais possíveis respostas, mas na busca de uma resposta própria e adequada. Esse processo pode ocorrer a partir da deliberação moral como uma forma de analisar as situações a auxiliar os enfermeiros nas tomadas de decisões. No desempenho deliberativo por meio do desenvolvimento da competência ética, há a expectativa em ser ator na tomada de decisão, o que significa agir e comprometer-se na relação de cuidados, respeitando os desejos e os direitos da pessoa cuidada.

E, por fim, o Poder Público precisa implementar políticas públicas efetivas para transformar essa realidade: empregar de forma racional os recursos públicos para aumentar os investimentos e ampliar a quantidade de vagas em UTI pelo SUS e credenciar mais leitos em hospitais particulares. Dessa forma, o direito à saúde será oferecido de forma digna, conforme está previsto em lei.

As limitações deste estudo podem estar relacionadas ao método empregado, pois não é possível determinar a probabilidade de seleção de cada participante na pesquisa e a discussão dos resultados com a literatura. Como é escassa a produção científica acerca da temática, os achados da pesquisa foram analisados, considerando o detalhamento de cada resultado, com atuais estudos nacionais e internacionais. Logo, as percepções e particularidades da organização profissional da enfermagem e da estrutura dos sistemas de saúde nos países de origem dos respectivos estudos influenciam, também, a discussão dos dados. Contudo, aponta-se que há um entendimento de que as demandas relacionadas à prática no contexto da terapia intensiva assemelham-se em diferentes países; o que diverge de outros países é justamente a questão da internação por ordem judicial. Assim sendo, sugere-se a realização de novas pesquisas visando o aprofundamento da questão em estudo, direcionando analisar os problemas éticos vivenciados por enfermeiros em situações de internação por ordem judicial na perspectiva da deliberação moral em diferentes cenários. 


\section{Referências}

1 Oliveira MRM. A judicialização da saúde no Brasil. Rev Tempus Actas Saúde Col. [Internet]. 2013 [acesso em 2018 jul 03]; 7(1): 79-90. Disponível em: http://www.tempusactas.unb.br/index.php/tempus/article/view/1276doi: http://dx.doi.org/10.18569/tempus.v7i1.1276

2 Ramos RS, Gomes AMT. A judicialização da saúde pública no Brasil: um estudo de representações sociais. RevCuid. [Internet]. 2014 [acesso em 2018 Jul 03]; 5(2): 827-36. Disponível em: http://www.scielo.org.co/pdf/cuid/v5n2/v5n2a16.pdfdoi: http://dx.doi.org/10.15649/cuidarte.v5i2.124

3 Gomes FFC, Cherchiglia ML, Machado CD, Santos VC, Acurcio FA, Andrade EIG. Acesso aos procedimentos de média e alta complexidade no Sistema Único de Saúde: uma questão de judicialização. Cad Saúde Pública [Internet]. 2014 Jan [acesso em 2018 Jul 03]; 30(1): 31-43. Disponível em:http://www.scielo.br/pdf/csp/v30n1/0102-311X-csp-30-01-00031.pdfdoi: http://dx.doi.org/10.1590/0102-311X00176812.

4 Bittencourt GB. O "Estado da Arte" da produção acadêmica sobre o fenômeno da judicialização da saúde no Brasil. Cad Ibero-AmerDirSanit. Brasília [Internet]. 2016 Jan/mar [acesso em 2018 jul 03]; 5(1): 102-21. Disponível em: https://www.cadernos.prodisa.fiocruz.br/index.php/cadernos/article/view/261/329doi: http://dx.doi.org/10.17566/ciads.v5i1.261

5 Biehl J, Petryna A. Tratamentos jurídicos: os mercados terapêuticos e a judicialização do direito à saúde.Histcienc saúde-Manguinhos [Internet]. 2016 Jan/mar [acesso em 2018 jul 03]; 23(1): 173-92. Disponível em: http://www.scielo.br/pdf/hcsm/v23n1/0104-5970-hcsm-23-1-0173.pdfdoi: http://dx.doi.org/10.1590/S0104-59702016000100011.

6 Ramos RS, Gomes AMT, Oliveira DC, Marques SC, Spindola T, Nogueira VPF. Access the Unified Health System actions and services from the perspective of judicialization. Rev LatinoAm Enferm. [Internet]. 2016 [cited 2018 jul 03] 24: e2797. Available from: http://www.scielo.br/pdf/rlae/v24/pt_0104-1169-rlae-24-02797.pdfdoi: http://dx.doi.org/10.1590/1518-8345.1012.2689.

7 Vargas MAO, Ramos FRS, Schneider DG, Schneider N, Santos AC, Leal SMC. Hospitalization by court order: ethical dilemmas experienced by nurses. Rev GaúchaEnferm. [Internet]. 2013 Mar [cited 2018 Jul 04]; 34(1): 119-25. Available from: http://www.scielo.br/pdf/rgenf/v34n1/en_15.pdfdoi: http://dx.doi.org/10.1590/S198314472013000100015. 
8 Schneider DG, Ramos FRS. Moral deliberation and nursing ethics cases: elements of a methodological proposal. Nurs Ethics. [Internet] 2012 Nov [cited 2018 jul 04]; 19(6): 764-76. Available from:https://www.ncbi.nlm.nih.gov/pubmed/22619237 doi: 10.1177/0969733011420096.

9 Nora CRD, Deodato S, Vieira MMS, Zoboli ELCP. Elements and strategies for ethical decisionmaking in nursing. Texto context enferm. [Internet]. 2016 [cited 2018 Jul 04]; 25(2): e4500014. Available from: http://www.scielo.br/pdf/tce/v25n2/0104-0707-tce-25-02-4500014.pdfdoi: http://dx.doi.org/10.1590/0104-07072016004500014.

10 Biernacki P, Waldorf D. Snowball Sampling: Problems and techniques of Chain Referral Sampling.Sociol Methods Res. [Internet] 1981 [cited 2018 Jul 04]; 10(2): 141-63. Available from:http://journals.sagepub.com/doi/abs/10.1177/004912418101000205?journalCode=smra doi: https://doi.org/10.1177/004912418101000205

11 Moraes R, Galiazzi MC. Análise textual discursiva. 2 ed. EditoraUnijuí; 2016. 264 p.

12 Vargas MAO, Ramos FRS. Autonomia na unidade de terapia intensiva: comecemos por cuidar de nós. Revbrasenferm. [Internet]. 2010 Dez [acesso em 2018 Jul 04]; 63(6): 956-63. Disponível em: http://www.scielo.br/pdf/reben/v63n6/14.pdf

13 Schaefer R, Vieira M. Ethical competence as a coping resource for moral distress in nursing. Texto context enferm. [Internet]. 2015 Jun [cited 2018 Jul 04]; 24(2): 563-73. Available from: http://www.scielo.br/pdf/tce/v24n2/0104-0707-tce-24-02-00563.pdfdoi: http://dx.doi.org/10.1590/0104-07072015001032014.

14 Luz KR, Vargas MAO, Schmidtt PH, Barlem ELD, Tomaschewski-Barlem JG, Rosa LM. Ethical problems experienced by oncology nurses. Rev Latino-Am Enferm. [Internet]. 2015 Dec [cited 2018 Jul 04]; 23(6): 1187-94. Available from: http://www.scielo.br/pdf/rlae/v23n6/0104-1169-rlae23-06-01187.pdfdoi: http://dx.doi.org/10.1590/0104-1169.0098.2665.

15 Barlem ELD, Lunardi VL, Tomaschewski JG, Lunardi GL, Lunardi FWD, Schwonke CRGB. Moral distress: challenges for an autonomous nursing professional practice. Rev esc enferm USP [Internet]. 2013 Apr [cited 2018 Jul 04]; 47(2): 506-10. Available from: http://www.scielo.br/pdf/reeusp/v47n2/33.pdfdoi: http://dx.doi.org/10.1590/S008062342013000200033.

16 Van der Dam S, Schols JM, Kardol TJ, Molewijk BC, Widdershoven GA, Abma TA. The Discovery of deliberation. From ambiguity to appreciation through the learning process of doing Moral Case Deliberation in Dutch elderly care. Soc Sci Med. [Internet]. 2013 Apr [cited 2018 Jul 04]; 83: 125-32. Available from: https://www.ncbi.nlm.nih.gov/pubmed/23410684doi: 10.1016/j.socscimed.2013.01.024 
17 Molazem Z1, Tavakol N, Sharif F, Keshavarzi S, Ghadakpour S. Effect of education based on the "4A Model” on the Iranian nurses' moral distress in CCU wards. J Med Ethics Hist Med. [Internet] 2013 Apr [cited 2018 Jul 04]; 6:5. Available from: https://www.ncbi.nlm.nih.gov/pmc/articles/PMC3740193/pdf/jmehm-6-5.pdf

18 Oerlemans AJ, Van Sluisveld N, van Leeuwen ES, Wollersheim H, Dekkers WJ, Zegers M6. Ethical problems in intensive care unit admission and discharge decisions: a qualitative study among physicians and nurses in the Netherlands. BMC Med Ethics. [Internet] 2015 Feb [cited 2018 Jul 04]; 16:9. Available from: https:/www.ncbi.nlm.nih.gov/pmc/articles/PMC4344998/pdf/12910_2015_Article_1.pdfdoi: 10.1186/s12910-015-0001-4

19 Andrade RD, Mello DF, Silva MAI, Ventura CAA. Advocacia em saúde na atenção à criança: revisão da literatura. Revbrasenferm. [Internet]. 2011 Ago [acesso em 2018 Jul 04]; 64(4): 738-44. Disponível em: http://www.scielo.br/pdf/reben/v64n4/a17v64n4.pdfdoi: http://dx.doi.org/10.1590/S0034-71672011000400017

20 Rodríguez-Carvajal M, Mora D, Doblas A, García M, Domínguez P, Tristancho A, et al. Impacto de las altas no programadas en la mortalidad hospitalaria tras la estancia en una unidad de cuidados intensivos. Med Intensiva [Internet]. 2011 Abr [acesso em 2018 Jul 04]; 35(3): 143-49. Disponibleen: http://scielo.isciii.es/pdf/medinte/v35n3/original1.pdf

21 Rates CMP, Pessalacia JDR. Enfermagem brasileira frente às questões bioéticas: revisão integrativa. RevEnfermCent O Min. [Internet] 2014 jan/abr [acessoem 2018 Jul 05]; 4(1): 1036-47. Disponível em: http://www.seer.ufsj.edu.br/index.php/recom/article/view/567/578doi: http://dx.doi.org/10.19175/recom.v0i0.567

22 Nora CRD, Zoboli ELCP, Vieira MM. Ethical deliberation in health: an integrative literature review. Rev Bioét. [Internet]. 2015 Apr [cited 2018 Jul 04]; 23(1): 114-23. Available from: http://www.scielo.br/pdf/bioet/v23n1/en_1983-8034-bioet-23-1-0114.pdfdoi:

http://dx.doi.org/10.1590/1983-80422015231052

23 Vargas-Peláez CM, Rover MR, Leite SN, Rossi Buenaventura F, Farias MR. Right to health, essential medicines, and lawsuits for access to medicines - a scoping study. Soc Sci Med. [Internet] 2014 Nov [cited 2018 Jul 05]; 121: 48-55. Available from: https://www.ncbi.nlm.nih.gov/pubmed/25306409doi: 10.1016/j.socscimed.2014.08.042

24 Wiegand DL, Grant MS, Cheon J, Gergis MA. Family-centered end-of-life care in the ICU. J GerontolNurs. [Internet] 2013 Aug [cited 2018 Jul 05]; 2013 Aug; 39(8): 60-8. Available from: https://www.ncbi.nlm.nih.gov/pubmed/23758114doi: 10.3928/00989134-20130530-04 
Deliberação moral de enfermeiros frente à internação por ordem judicial | 20

\section{Autor correspondente}

Kely Regina da Luz

E-mail: kelydaluz@yahoo.com.br

Endereço: R. Ramiro Barcelos, 2350

CEP: 90035-007

\section{Contribuições de Autoria}

Nome 1 - Kely Regina da Luz

Contribuições: concepção, planejamento, análise e a interpretação dos dados.

Nome 2 - Mara Ambrosina de Oliveira Vargas

Contribuições: orientação na concepção, planejamento, análise e a interpretação dos dados

Nome 3 - Edison Luiz Devos Barlem

Contribuições: orientação na concepção, planejamento, análise e a interpretação dos dados

Nome 4- Dulcinéia Ghizoni Schneider

Contribuições: revisão crítica do conteúdo e participação da aprovação da versão final do manuscrito

Nome 5 - Franciele Budziareck das Neves

Contribuições: revisão crítica do conteúdo e participação da aprovação da versão final do manuscrito

\section{Como citar este artigo}

Luz KR, Vargas MAO, Barlem ELD, Schneider DG, Neves FB. Deliberação moral de enfermeiros frente à internação por ordem judicial. Rev. Enferm. UFSM. 2019 [Acesso em: 2019 jun 15];vol 9 ex:1-20. DOI:https://doi.org/10.5902/2179769235033 